Sharif University of Technology
Scientia Iranica
Transactions E: Industrial Engineering
http://scientiairanica.sharif.edu
IRAENTIA

\title{
The perils of group purchasing in a competing supply chain
}

\author{
M. Soleimani Sedehi ${ }^{a}$, A. Makui ${ }^{a}, *$, and E. Bolandifar ${ }^{b}$ \\ a. Department of Industrial Engineering, Iran University of Science and Technology, Tehran, Iran. \\ b. Department of Decision Sciences and Managerial Economics, the Chinese University of Hong Kong, Hong Kong.
}

Received 8 April 2017; received in revised form 2 June 2018; accepted 24 September 2018

\section{KEYWORDS}

Purchasing strategy;

Multiunit bilateral

bargaining;

Market competition;

Quantity discount;

Wholesale price

contract.

\begin{abstract}
Group Purchasing Organizations (GPOs) are well-known intermediary firms that play an important role in some supply chains. An important question that arises regarding GPOs is whether a GPO that benefits from group buying discounts always benefits Original Equipment Manufacturers (OEMs) under market competition. In other words, does the role of a GPO always result in a win-win outcome for OEMs and GPOs? In response, a bargaining framework has been used to investigate the procurement strategies of competing OEMs. The incorporation of a GPO in a two-tier supply chain consisting of two competing OEMs with a common supplier that has a quantity discount menu is analyzed. The result shows that low-purchasing cost for GPOs may harm OEMs from a cost-benefit perspective. This unintuitive result can be explained by different impacts that a GPO has on the purchasing process. Although a GPO can enlarge the size of trade surplus, it has important influence on the size of the slice of the pie (profit sharing). Moreover, the procurement strategy of an OEM in equilibrium depends on not only the bargaining power, but also the competing OEM. Interestingly, unlike a weak OEM, a strong one may not prefer procuring through GPO.
\end{abstract}

(C) 2019 Sharif University of Technology. All rights reserved.

\section{Introduction}

Owing to the economic evolution in recent years, the emergence of complex international supply chains with worldwide manufacturers, usually called Original Equipment Manufacturers (OEMs), and also the strong role of intermediaries in supply chains because of their constructive role in the efficiency of supply chains have become very important. The notion of intermediary in economics literature represents those economic agents

\footnotetext{
*. Corresponding author. Tel.: +9821 73913004 ;

Fax: +982189779415

E-mail addresses: msoleimani@iust.ac.ir (M. Soleimani

Sedehi); amakui@iust.ac.ir (A. Makui);

ehsan@baf.cuhk.edu.hk (E. Bolandifar)
}

doi: $10.24200 /$ sci.2018.21043 that coordinate and arbitrate transactions between a group of supply chain firms [1]. The significance of this notion led to Spulber's proposition of the intermediation theory of the firm [2] in 1996. He believes that an intermediary acts as the fundamental building block of economic activities.

Group Purchasing Organizations (GPOs) are well-known intermediary firms that play an important role in some supply chains (especially in retail and healthcare supply chains). The role of GPOs for buyers brings about many advantages. However, the fundamental rationale for joining a GPO is that a buyer firm will undergo lower total purchasing cost by purchasing through a GPO rather than purchasing directly from a supplier (because of demand aggregation on a larger scale). Although the cost-reduction benefit of group purchasing is well understood, its effect on buyers' 
performance is not so transparent. On the one hand, GPO's cost efficiency may bring savings for OEMs. On the other hand, the OEMs should share a part of their profits with the GPO and, naturally, profit allocation depends on the negotiated agreements.

This paper attempts to shed some light on when competitors can cooperate in purchasing and also what the perils of group purchasing are for them. Our study offers another possible explanation of why some OEMs may not join purchasing groups.

In a stylized two-tier supply chain system, two competing OEMs that have the option to procure directly from a supplier or to use a GPO to delegate their procurement are studied. To ensure a delegation agreement, each OEM engages in a bilateral negotiation with GPO. OEMs subsequently compete in the product market by setting quantities and using an often-used wholesale-price contract form in negotiations.

The endogeneity of reservation profits in the proposed problem highlights the importance of modeling firm negotiations under competition.

In this study, OEMs have four important characteristics that represent the real environment. First, each OEM seeks to maximize its total profit, not merely minimizing the purchasing costs. Second, each OEM's product demand, denoted by its purchasing requirement, affects its product price in the market. Third, OEMs that agree on purchasing through GPO are not forced to purchase only through GPO; instead, they can purchase directly, too. Fourth, OEMs determine their order quantities after deciding on the purchasing strategy, meaning that competition for orders precedes procurement.

The final assumption may require much clarification. In fact, firms compete and secure customer orders before negotiating contracts to procure inputs to fill those orders [3]. Indeed, the competition stage preceding the vertical negotiation stage would be applicable to environments, where input supply terms can be changed or renegotiated more frequently than customer orders (for instance, for durable goods or when customers are relatively patient) [3]. One well-known example is the supply chain management practice of Dell that has adopted a procurement strategy based on the following principle: "order from suppliers only when you receive demand from customers". Similar practices are common in some other industries such as building and architectural contracts, large-scale services for governments, and electricity and gas retailing. This approach is usually relevant when downstream firms in a supply chain find it more convenient to remain committed to contracts with customers than be locked into input supply contracts.

One important question to address is whether a GPO that benefits from group buying discounts is always beneficial to OEMs (their purchasing process) under constant competition and negotiations. To answer this critical question, a game theoretic model is developed that includes a common supplier with a quantity discount schedule, a profit-maximizing GPO that negotiates with OEMs to derive a purchasing contract, and two profit-maximizing OEMs that compete in a common market. In response to this question, a trade-off between two conflicting effects that results in the purchasing decision of an OEM is required. On the one hand, an OEM can potentially enjoy cost savings because of more quantity discount obtained from aggregated ordering using a GPO. On the other hand, the OEM must share part of its profit with the GPO for the purchasing service. Naturally, profit-sharing mechanisms depend on two main bargaining factors. One is the bargaining power of negotiators against each other; the second one is the bargaining position of the OEM and the GPO as well as their outside payoffs (i.e., their profits in the case of negotiation breakdown). The OEM's outside payoff is its profit when procuring directly from the supplier.

Analysis of OEM-GPO negotiations highlights the strategic perils of group purchasing, although it is always preferable for symmetric OEMs to use a GPO, and yet it may cause competing asymmetric OEMs to worse off and leads to a win-lose outcome, in which the GPO gains and some OEMs lose. In some conditions, OEM with lower bargaining power may obtain less profit and, thus, may be less likely to purchase through the GPO than that with higher bargaining power. These results are derived from the analysis of the incorporation of a GPO in a two-tier supply chain that consists of two competing OEMs with a common supplier. A bilateral bargaining framework is used to model negotiations of wholesale price contracts.

The sequence of events in the proposed model is as follows. First, OEMs determine their purchasing strategy whether using a GPO or direct purchasing. The decision is already known for all firms. Second, OEMs compete in the market and receive orders from consumers. Third, based on their known order quantities, OEMs procure their orders from a supplier. If they agree on using a GPO, they make contracts with GPO in a bargaining framework. If not, they procure directly from the supplier. In the former case, the wholesale price is determined based on bargaining; in the latter one, the wholesale price is determined based on a predefined quantity discount schedule.

The model is analyzed within two structures: a symmetric case (OEMs with equal bargaining power) and an asymmetric case (OEMs with different bargaining power).

The remainder of this paper is organized as follows. A review of the relevant literature is presented in Section 2. The model is introduced in Section 3. Extracting some insights and results based on the 
model analysis is presented in Section 4. Section 5 presents the conclusion, and all proofs are relegated to the appendix.

\section{Literature review}

This paper attempts to integrate three main research streams in the literature of supply chain management (also called channel distribution literature in marketing) into one river. Those different streams include competition, contracting (bargaining), and quantity discount. This integrated river is the procurement strategy.

Considering the competition stream, Ingene and Parry [4] were the first to introduce competing retailers to the quantity discount literature. Our model is constituted based on deterministic demands and price competition; as Tsay and Agrawal [5] pointed out, due to model complexity, typically deterministic formulations are found in most existing multi-echelon analyses that incorporate demand/price competition.

The second stream of related researches addresses the implication of bargaining on the allocation and level of supply chain profits. As is the case in our paper, some recent studies have considered bargaining contracts rather than take-it-or-leave-it price offers [611], which has been the case in previous models of channel interaction [12]. As Lovejoy [13] pointed out, a bargaining model would be more appropriate in many supply chain contexts, because the solutions derived from the Stackelberg framework can be highly impractical because of various issues.

Feng and $\mathrm{Lu}$ carried out a number of novel researches [8-10] on production outsourcing. They were the first researchers to have investigated market competition and contract bargaining concurrently with respect to an outsourcing problem. They have investigated market competition and vertical contracting in a two-tier supply chain consisting of two manufacturers and one/two suppliers. Although their researches share certain similarities with this study, there are some significant differences. First, the main focus is on the role of an intermediary (like GPO) in the procurement strategy, while their researches focus on the outsourcing strategy. Second, the production (procurement) cost is constant in their studies; however, it is related to order quantity in our model (using quantity discount). Third and more importantly, in their problem setting, downstream firms compete in the market after determining the wholesale prices under contract bargaining. In contrast, early ordering (competition before negotiation) is used in our problem definition. Therefore, although our results confirm part of their results, the results of this paper show some more new findings.

Feng and $\mathrm{Lu}[10]$ carried out research on out- sourcing most relative to our study that considers both negotiation and competition in a two-tier supply chain. They investigated whether low-cost outsourcing always is beneficial to downstream manufacturers. They considered a two-tier supply chain with one common supplier and two competing manufacturers that have different fixed producing costs. They showed that low-cost outsourcing might lead to a win-lose outcome whose suppliers gain and the manufacturers lose. Due to bargaining externality (derived from different bargaining positions), they concluded that as the manufacturer's bargaining power decreases, his/her profit under outsourcing may increase and may be more likely for her to outsource. In some cases, manufacturers with higher bargaining power may obtain lower outsourcing profit and, thus, may be less likely to outsource than those with lower bargaining power (in contrast with our results). Our research has some important differences with that of Feng and $\mathrm{Lu}[10]$ :

- They do not use any quantity discount in their model, and all costs are fixed and predefined;

- In their problem setting, the negotiation stage precedes the competition stage. However, in our study, market quantities and prices are determined before negotiation;

- They use a revenue-sharing contract format in negotiations (assuming fixed centralized profit); however, a wholesale price contract format has been implemented in this paper that is more general in the real world, yet more complicated.

There is a vast operations and supply chain management literature on contracting; however, existing literature on GPOs and other contracting intermediaries is limited and still developing. Assuming a manufacturer that offers a linear quantity discount to competing retailers, Chen and Roma [14] identified the conditions under which a GPO would be formed. They were the first to study group buying in a competing distribution channel. They studied a supply chain with a monopoly manufacturer that offers a linear quantity discount to two competing retailers. They identified the conditions under which a GPO would form when the retailers' demands were the functions of retail prices in the market. Unlike our paper, they did not consider bargaining in their model. Similar to the result of the current study, yet in different conditions, they showed that downstream firms might get hurt by group purchasing. In their paper, group buying is always preferable for symmetric retailers in the market. However, under the asymmetric case, it can be detrimental to larger retailers. However, in our paper, downstream firms are always symmetric in the market; yet, they may differ in their bargaining power with a GPO. In this study, group purchasing can be 
detrimental to the weaker OEM, which is in contrast with the finding of Chen and Roma [14].

The existing literature on group purchasing apparently has not modeled firm negotiations in a competitive setting, thereby cannot provide insights into this important trade-off in competing OEMs' purchasing decisions. Specifically, most studies that have implemented a GPO in the supply chain assume that GPO's interests are aligned with buyers; thus, the GPO seeks to minimize the buyers' total purchasing costs [15-17]. All of these papers use a take-it-orleave-it negotiation format if necessary. More recently, $\mathrm{Hu}$ et al. [18] studied the role of a GPO as an independent entity in a healthcare supply chain that tries to maximize its own profit. Their study along with their previous study [16] provides the first theoretical analyses of healthcare GPOs. They studied the impact of GPOs on healthcare-product supply chains [18]. They investigated how the presence of a GPO affected the buyers' total purchasing costs in a supply chain with one common upstream supplier and two heterogeneous buyers (and $n$ identical buyers). Although there are some similarities between the structure of the supply chain in our paper and that of the former paper, there are noticeable differences between these two research studies. The most important difference is that no competition is considered between buyers in that paper, and all demands are fixed in advance. However, the demands are determined in a competing market in our paper. In addition, there is no negotiation between firms, and each firm tries to maximize its profit and minimize its cost individually.

Early ordering is another characteristic of our model that differentiates it from previous studies. There are few studies in the literature that have modeled competition for orders prior to procurement negotiations. In an apparently pioneering study in its own kind, Stahl [19] assumed that bidding for inputs took place after the downstream competition for forward contracts, leading to competitive outcomes across the entire vertical chain. Our study is different from his model, since he sets a single input price (there is no price discrimination in the wholesale negotiation) and downstream firms have all the power in that market. In contrast, our model presumes that the single upstream firm (GPO) bargains bilaterally with each of the downstream firms.

In a more relevant study, Gans [20] studied vertical contracting when competition for orders preceded procurement. According to his study, when competition for orders precedes negotiations for component procurement, the outcome is the oligopolistic competitive outcome (i.e., Cournot competition outcome). He also studies the impact of vertical integration on this outcome. Although he considers a non-decreasing supplying cost with respect to order quantities, a more realistic and often-used non-increasing supplying cost, called quantity discount model, is used here. Gans considered a two-tier supply chain; however, our main focus is on a three-tier supply chain.

More recently, Guo and Iyer [11] studied a multiunit bilateral bargaining and downstream competition in a two-tier supply chain where market competition takes place before ex-post negotiations. Their study is focused on bargaining timing and determines the firms' equilibrium decision whether bargaining concurrently or sequentially.

Briefly, this study generalizes the existing knowledge on GPO by considering a number of structural features:

(i) Quantity discount model for supplying the product;

(ii) Product substitutability;

(iii) Firms' competition;

(iv) Bargaining powers (which have been highly investigated in the literature) in one framework, simultaneously.

The question is: Why did most previous papers consider two or three of these four features in one research framework?

\section{The proposed model}

A well-known supply chain framework is considered consisting of one common supplier who sells homogeneous goods based on a quantity discount menu to two OEMs (indexed by $i, j=1,2$ ) that compete in a common market by selling goods to end consumers. A quantity discount menu, in fact, represents a competitive supply market where there are many suppliers that produce a general item. OEMs' products are substitutable in the market and consist of the main component procured from a supplier market. Without loss of generality, OEMs' production cost is assumed to be equal to zero. Therefore, the procurement cost constitutes the main part of the market price.

In the initial market equilibrium, each OEM acts individually in procuring from the supplier. Then, the OEMs consider the option of procuring through a GPO to obtain a lower wholesale price. This option is possible only when both OEMs agree to cooperate.

\subsection{Market competition}

The demand quantity for each product in the market is elastic. For tractability, a Cournot model is used and known as an often-used linear price model.

$$
\mathbf{p}_{\mathbf{i}}=\alpha-\mathbf{q}_{\mathbf{i}}-\gamma \mathbf{q}_{\mathbf{j}}
$$

where $i \in\{1,2\}$ and $j=3-i$. $\mathbf{p}_{\mathbf{i}}$ and $\mathbf{q}_{\mathbf{i}}$ show the market price and quantity, respectively. $\alpha$ is the initial 
market price, and $\gamma \in(0,1)$ measures the degree of product substitutability. When $\gamma$ approaches 0 , the two products become independent, and there will be no competition in the market. When $\gamma$ approaches 1 , they become perfect substitutes. As implied by Relation (1), both OEMs are symmetric in the market.

\subsection{Procurement process}

It is assumed that OEMs first compete in the market for orders and, then, procure their needs based on their procurement strategy. Here, they have two alternatives for their procurement strategy: whether to procure directly from a common supplier (abbreviated by DP) or procuring through a GPO (abbreviated by GPO). According to the Robinson-Patman act, a common price menu must be offered to each buyer to preclude sellers "from giving different terms to different resellers in the same reseller class." Therefore, the same price menu is offered under either individual or group purchase.

In our problem setting, for the sake of analytical simplicity and tractability, the supplier (indexed by $s$ ) offers a linear quantity discount schedule, Relation (2), which satisfies two important practical situation conditions: non-increasing volume discount and nondecreasing total purchasing cost.

$$
w_{s i}=\lambda-\mu q_{i}
$$

where $i \in\{1,2, c\}$, and $w_{s i}$ shows the wholesale price offered from the supplier to OEM $(i)$ or GPO (indexed by $c$ ). $\lambda$ is the basic wholesale price (when quantity is zero), which is normalized to 1 without loss of generality, and $\mu$ stands for discount rate. Although $\mu$ can vary at the interval $[0,1]$ theoretically, we do not have discount rates higher than $\frac{1}{2}$ in practice. Since $\mu \leq 1 / 2$ simplifies the analytic process in this paper, it is assumed that $\mu$ is less than $\frac{1}{2}$.

\subsection{Bargaining and contracts}

To model negotiations in the procurement through the GPO, a multi-unit bilateral bargaining framework is used to model contract negotiations. Each OEM negotiates bilaterally with a common GPO over a wholesale price. This bilateral formulation of negotiations reflects the fact that competing OEMs typically negotiate independently. In a competition, the bargaining outcome of one channel naturally depends on that of the other. When procuring through the GPO, OEMs negotiate with the GPO to determine the wholesale price that they must pay to the GPO. To model these bilateral negotiations, the asymmetric Nash bargaining solution is used.

$$
\max _{w_{i}} \phi_{i}=\left(\pi_{i}\left(w_{i}\right)-\delta_{i}\right)^{\theta_{i}}\left(\pi_{c}\left(w_{i}\right)-\delta_{c i}\right)^{\left(1-\theta_{i}\right)},
$$

where $\phi_{i}$ is the Nash bargaining product, $w_{i}$ is the wholesale price offered by the GPO to OEM $(i)$, and $\pi_{i}$ is the firm $i$ 's profit. When OEM $(i)$ and the GPO negotiate, $\delta_{i}$ and $\delta_{c i}$ stand for OEM $(i)$ 's and the GPO's reservation profits (also called disagreement point or outside option in the literature, i.e., the firm's profit when no agreement is achieved), respectively. $\theta_{i} \in[0,1]$ denotes OEM $(i)$ 's bargaining power vis-à-vis the GPO.

The negotiation between OEM $(i)$ and the GPO is considered over the wholesale price of product $i$. Two possible outcomes arise from the negotiation:

1. The trade agreement fails and the OEM procures directly from the supplier, or:

2. An agreement is reached, and the GPO procures the product for the OEM.

To define the bargaining problem, disagreement points of the trading parties should be specified, i.e., their profits when the negotiation breaks down $\left(\delta_{i}\right.$ and $\left.\delta_{c i}\right)$.

The value of a negotiator's outside option is referred to as its "bargaining position." This is distinguished from the "bargaining power," which represents the relative skill of negotiators.

The negotiation outcomes of the two competing supply chains form Nash equilibrium. This solution concept for our multi-unit bilateral bargaining problem is known as the Nash- solution. This approach is viewed as a direct extension of the single-unit Nash bargaining solution to multiple bargaining units [10].

\subsection{Sequence of events}

At first, the OEMs determine their procurement strategy: whether to procure directly from a component supplier or through the GPO. If they decide to procure directly, they determine their order quantities in the market and, then, procure their orders from the supply market independently. In this strategy, the wholesale prices are determined based on a pre-defined quantity discount schedule. If they decide to use a GPO for component procurement, then:

1. The OEMs compete in the product market to determine their order quantities;

2. A contract is negotiated bilaterally between each of the OEMs and the GPO to determine the wholesale price of the component. All negotiations occur in parallel. Upon reaching an agreement, the OEM contracts with the GPO to procure her demand; otherwise, the OEM procures directly from the supplier;

3. The OEMs place an order from the GPO (or directly procure from the supply market). The GPO procures from the supply market and charges the OEMs' negotiated prices.

\subsection{Assumptions}

- To focus on the competition and negotiation's effects on optimal procurement structure, all model param- 
eters are assumed to be deterministic and common knowledge;

- Zero coordination cost under group purchasing;

- $\mu \leq 1 / 2$. This is a rational condition that occurs in reality most of the times and helps us with analytical proofs;

- OEMs are similar in the market and may differ only in their bargaining power;

- Without loss of generality, $\theta_{1} \leq \theta_{2}$;

- The supplier offers a quantity discount schedule that is non-increasing in quantity and ensures nondecreasing revenue;

- For simplicity, the OEMs' production cost is zero. This is true when equal production costs are considered for both OEMs;

- All firms have common knowledge under complete information;

- The OEMs, GPO, and supplier are risk neutral: each seeks to maximize its own expected profit.

\subsection{Notations}

Tables 1 and 2 show a snapshot of parameters and variables, respectively.

\section{Analysis}

In this section, the behavior of firms under different procurement strategies is investigated analytically. Since there are numerous parameters that make it difficult to study the equilibrium behaviors, the problem is investigated in two symmetric and asymmetric cases with respect to equal or different bargaining powers, respectively. Since these two cases differ only on OEMs' bargaining powers situation, they differ only under GPO procurement strategy, while they are alike in the case of DP strategy.

\subsection{Symmetric case}

The symmetric case provides a condition when both OEMs have the same bargaining powers vis-à-vis a GPO $\left(\theta=\theta_{1}=\theta_{2}\right)$. In this case, both OEMs have similar behavior under GPO strategy similar to what occurs under DP strategy. As a matter of fact, there is just one bilateral monopoly problem when both OEMs are identical. As shown in Proposition 1, GPO is the dominant strategy in the symmetric case.

Proposition 1 (equilibrium strategy). When OEMs have equal bargaining power, then GPO strategy is the best decision for all supply chain players in equilibrium.

The intuition behind this proposition is straightforward and is considered as the most important motive for group purchasing $[10,14]$. It is clear that the GPO always benefits from group purchasing strategy, because he obtains zero profit otherwise. For analyzing the behavior of OEMs in equilibrium, it should be mentioned that the order quantities (accordingly, market prices) are determined before bargaining over the wholesale prices. When procuring through GPO, OEMs benefit from larger order quantities by more discounts. This enlarges the size of the pie (trade surplus) to be shared between OEMs and GPO. Since OEMs have larger order quantities under GPO, their market prices will decline. Consequently, both of the

Table 1. Summary of parameters.

\begin{tabular}{cl}
\hline Parameter & \multicolumn{1}{c}{ Definition } \\
\hline$\alpha$ & The basic price of a product in the market \\
$\gamma$ & The substitution effect of products \\
$\lambda$ & The basic price of the key component (with no discount) \\
$\mu$ & Quantity discount rate offered from the supplier \\
$\theta_{i}$ & OEM $(i)$ 's bargaining power vis-a-vis GPO \\
\hline
\end{tabular}

Table 2. Summary of variables.

\begin{tabular}{cl}
\hline Variable & \multicolumn{1}{c}{ Definition } \\
\hline$q_{i}$ & The demand for OEM $(i)^{\prime}$ 's product in the market \\
$p_{i}$ & The price for OEM $(i)^{\prime}$ 's product in the market \\
$w_{s i}$ & The wholesale price of the key component offered from the supplier to OEM $(i)$ \\
$w_{c i}$ & The wholesale price of the key component offered from GPO to OEM $(i)$ \\
$w_{s c}$ & The wholesale price of the key component offered from the supplier to GPO \\
$\pi_{i}$ & The profit of firm $i$ \\
$\delta_{i}$ & The reservation profit of firm $i$
\end{tabular}


lower wholesale price that the GPO should pay to the supplier (because of higher order quantity) and the lower market price for OEMs force the negotiated wholesale price to decrease when bargaining. It is simply shown that each OEM's margin $\left(p_{i}-w_{i}\right)$ is larger under GPO compared to DP, and it is obvious that OEMs have larger order quantities under GPO, too. As a result, there is higher profit for each OEM under GPO compared to DP. Therefore, GPO strategy is a win-win strategy for both GPO and OEMs.

Since GPO procurement strategy is the equilibrium decision in this game, in the following propositions, the role of different parameters in decision variables under GPO strategy is further investigated.

Proposition 2 (effect of bargaining power). Under GPO strategy, a higher bargaining power (higher $\theta)$ leads to:

\section{i) higher market demand;}

ii) lower market price;

iii) lower wholesale price;

iv) higher profits for OEMs;

v) lower profit for GPO.

Following an early ordering mechanism, the OEM knows that her share of the pie (trade surplus) increases in her bargaining power. Therefore, she tries to enlarge the pie when her bargaining power increases. In this respect, she makes a larger order quantity. Based on Relation (1), larger order quantity results in lower market price and lower wholesale price, consequently.

Most importantly, the OEM's bargaining power has positive effect on OEM's profit and negative effect on GPO and contract profits, intuitively. To further understand the effect of OEM's bargaining power on firms' profits, the firms' and the contractor's profits (total profit of both sides of the negotiation) are plotted as a function of the OEM's bargaining power in Figure 1. In this way, findings (iv) and (v) of Proposition 2 are supported. The profit behaviors shown in Figure 1 are similar for different $\alpha$ and $\gamma$.
It is also interesting from a managerial viewpoint to investigate the impact of product competition intensity on decision variables, shown in the next proposition.

Proposition 3 (effect of competition intensity). A higher competition level $(\gamma)$ leads to:

i) Lower market demand;

ii) Higher/lower market price;

iii) Higher wholesale price;

iv) Lower profits for OEMs;

v) Lower profit for GPO.

The effect of competition intensity in the market is not intuitive and needs more attention. Based on most of the previous studies $[8,14]$, competition intensity $(\gamma)$ is known to exert negative impact on market price and positive impact on market demand. However, here, there is an unusual impact, and assuming all other parameters fixed, higher competition intensity $(\gamma)$ always causes lower market demand.

Another interesting result is that although higher market competition causes higher wholesale prices that OEMs pay to GPO, a decrease in the GPO's profit will ensue. This unintuitive result is obtained because higher competition intensity exerts negative impact on market demand and, consequently, the size of the pie decreases. Smaller size of the pie reduces the profits of OEMs and GPO.

In addition, the effect of competition intensity on wholesale price is positive; however, its effect on market price is dependent on other parameters. Higher competition intensity $(\gamma)$ usually decreases market price (intuitive); yet, for $\frac{1}{4}<\mu<\frac{1}{2}$, there is a threshold for $\theta$ called $\tau_{1}(\mu)$ (this threshold has been defined in the Appendix.) according to which market price increases in $\gamma$ (unintuitive).

Focusing on more managerial decision variables (profits), the market competition has negative effects on both OEM's and GPO's profits as well as on contract profit. For better comprehension, the firms'

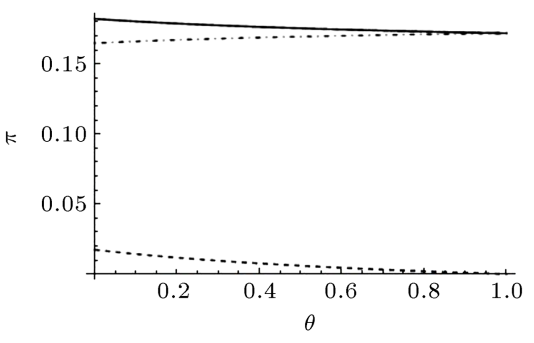

(a) Low discount $(\mu=0.05)$

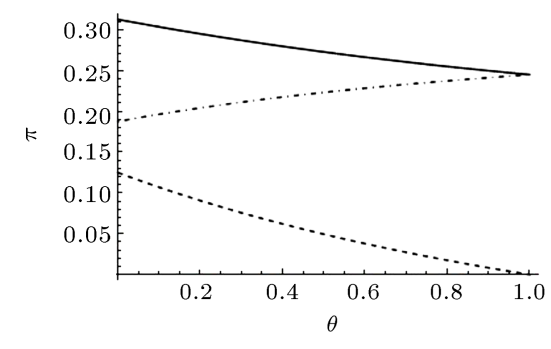

(b) Moderate discount $(\mu=0.25)$

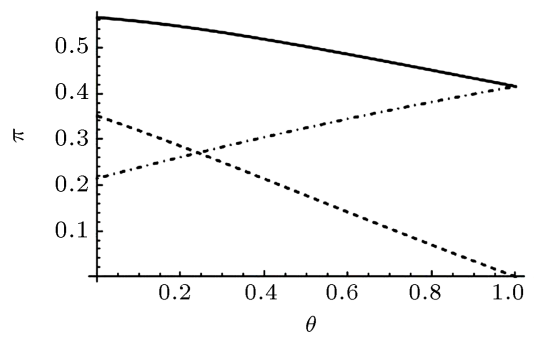

(c) High discount $(\mu=0.45)$

Figure 1. Effect of bargaining power on firms' profits under GPO strategy. Solid thick line: contract profit, solid line: OEM's profit, and dashed line: GPO's profit; $\alpha=2$ and $\gamma=0.5$. 


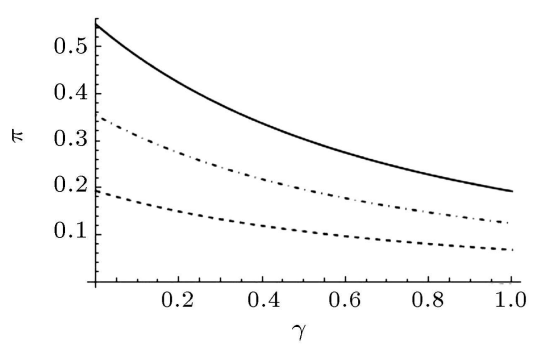

(a) Low bargaining power $(\theta=0.1)$

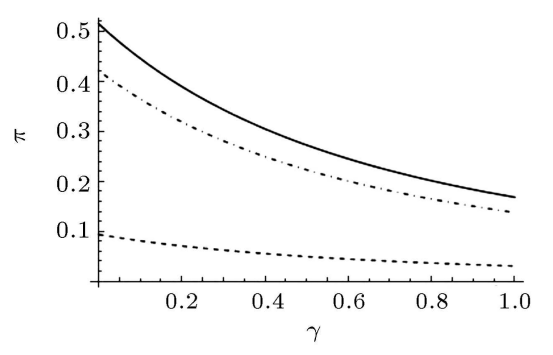

(b) Moderate bargaining power $(\theta=0.5)$

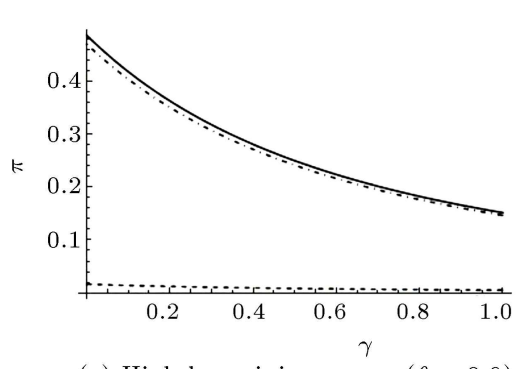

(c) High bargaining power $(\theta=0.9)$

Figure 2. Effect of competition intensity on firms' profits under GPO strategy. Solid thick line: contract profit, solid line: GPO's profit, and dashed line: OEM's profit; $\alpha=2$ and $\mu=0.25$.

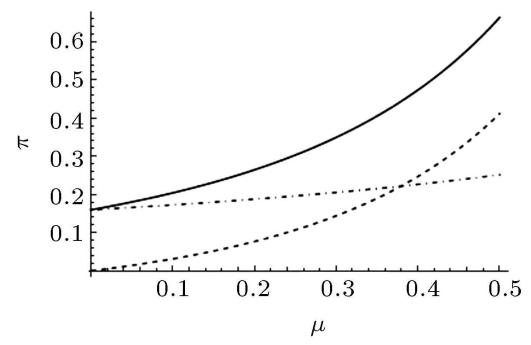

(a) Low bargaining power $(\theta=0.1)$

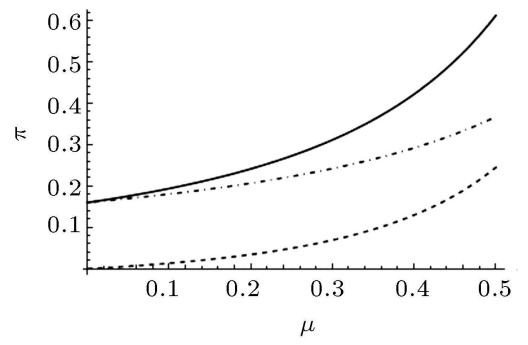

(b) Moderate bargaining power $(\theta=0.5)$

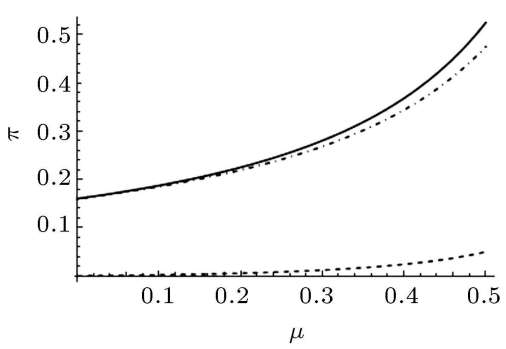

(c) High bargaining power $(\theta=0.9)$

Figure 3. Effect of quantity discount on firms' profits under GPO strategy. Solid thick line: contract profit, solid line: GPO's profit, and dashed line: OEM's profit; $\alpha=2$ and $\gamma=0.5$.

and the contractor's profits (the total profit of both sides of the negotiation) are plotted as a function of competition intensity in Figure 2. The profit behaviors shown in Figure 2 are similar for different $\alpha$ 's and $\mu$ 's.

Another important aspect of this study is the quantity discount schedule that suppliers offer. The impact of quantity discount rate on decision variables is investigated in the following proposition.

Proposition 4 (effect of quantity discount). higher quantity discount rate $(\mu)$ leads to:
i) Higher market demand;
ii) Lower market price;
iii) Lower wholesale price;
iv) Higher profits for OEMs;
v) Higher profit for GPO.

Assuming that all other parameters are fixed, a higher quantity discount rate $(\mu)$ always causes lower wholesale price for GPO and, consequently, lower wholesale price for OEMs. This lower wholesale price for OEMs leads to lower market price. Lower market price softens the competition in the market and causes higher market demand. Therefore, the size of the pie and the firms' profit increase. All of the results in Proposition 4 are intuitive.

Most importantly, the quantity discount rate has positive effect on both OEM's and GPO's profits as well as on the contractor's profit, because the higher the quantity discount rate, the larger the size of the pie.

To further understand the effect of quantity discount rate on firms' profits, the firms' and the contractor's profits are plotted as a function of the quantity discount rate in Figure 3. It supports findings (iv) and (v) of Proposition 4. The profit behaviors shown in Figure 3 are similar for different $\alpha$ 's and $\gamma$ 's.

Consequently, at the end of this subsection, the impact of important parameters on different decision variables (results of Propositions 2 to 4 ) is summarized in Table 3. From a consumer viewpoint, consumers always benefit when the bargaining power of OEMs or quantity discount rate (or both of them) increases.

Although most of the results in the symmetric case are intuitive, there are more challenging outcomes when the bargaining power of OEMs is different in the next subsection.

\subsection{Asymmetric Case}

In this case, the model is analyzed when retailers have different bargaining power. Without loss of generality, it is assumed that OEM1 has lower bargaining power than OEM2, when it negotiates with the GPO (i.e., $\left.\theta_{1} \leq \theta_{2}\right)$. The comparison of the two OEMs' profits indicates that both of them are identical when they directly procure goods from the supply market. It is also shown that OEM2 is better-off when OEMs procure through the GPO. 
Table 3. Changes of important variables when parameters become higher.

\begin{tabular}{lccc}
\hline & $\begin{array}{c}\text { Bargaining power } \\
(\boldsymbol{\theta})\end{array}$ & $\begin{array}{c}\text { Competition level } \\
(\gamma)\end{array}$ & $\begin{array}{c}\text { Quantity discount rate } \\
(\boldsymbol{\mu})\end{array}$ \\
\hline Market demand & Higher & Lower & Higher \\
Market price & Lower & Higher $/$ lower & Lower \\
Wholesale price & Lower & Higher & Lower \\
OEMs' profit & Higher & Lower & Higher \\
GPO's profit & Lower & Lower & Higher \\
Contract profit & Lower & Lower & Higher \\
\hline
\end{tabular}

Proposition 5 (OEMs' profits comparison). Under DP strategy, both OEMs are the same; however, when they procure through the GPO, the stronger OEM always:
i) Gets better-off;
ii) Offers lower prices in the market;
iii) Pays lower wholesale price;
iv) Receives larger order quantity in the market.

This proposition confirms the intuition that a stronger OEM benefits from its higher bargaining power, receiving lower prices from the GPO. Higher bargaining power of one of the OEMs might originate from different sources; for example, the OEM might have more expertise in procurement negotiations than the other OEM. This allows the OEM to get a better price in its contract from the GPO. As a result, it is able to offer more competitive prices in the market. In the first stage of the game, when OEMs compete over their order quantities, OEM2 characterized by its higher bargaining power, higher negotiation skills, and bargaining powers decides to be more aggressive in terms of increasing its order quantity and reducing its price.

Propostion 6 (equilibrium decisions). With different bargaining power, in equilibrium:

i) The stronger $O E M(O E M 2)$ prefers GPO to DP;

ii) The preferred procurement strategy of the weaker OEM depends on both OEMs' bargaining power;

iii) GPO always prefers GPO procurement strategy;

iv) The centralized profit in GPO procurement is greater than that in $D P$.

It is clear that the GPO always benefits from the group purchasing strategy, because he obtains no profit, otherwise. The stronger OEM (OEM2) also prefers GPO to DP since GPO strategy enlarges the trade surplus (size of the pie) and, also, increases her profit share (slice of the pie). The weaker OEM
(OEM1) prefers GPO to DP only when a threshold called:

$$
\tau_{2}=2(1-\mu)\left(\frac{1-\theta_{2}}{1-\theta_{1}}\right)\left(\frac{\theta_{1}}{\theta_{2}}\right)+2 \theta_{1}-\gamma \frac{1-\theta_{1} \theta_{2}}{1-\theta_{2}}
$$

is positive (this threshold that can be translated to $\Delta \theta$ is small enough), even if its bargaining power is either low or high. The interesting point about OEM1's behavior is that its tendency to GPO strategy is more dependent on its competitor bargaining power rather than its own bargaining power. If $\mu$ and $\gamma$ are considered fixed, OEM1's tendency towards GPO increases when both OEMs' bargaining power approaches $1 / 2$. In other words, the tendency for OEM1 to alter GPO strategy is shown when all participants (both OEMs and GPO) have similar bargaining power. This tendency decreases in product competition $(\gamma)$, while discount rate $(\mu)$ has insignificant effect on it. When $\Delta \theta$ becomes considerable, the weaker one prefers GPO to DP only when product competition $(\gamma)$ is very low.

Figure 4 shows a better sense of OEM1's behavior in equilibrium.

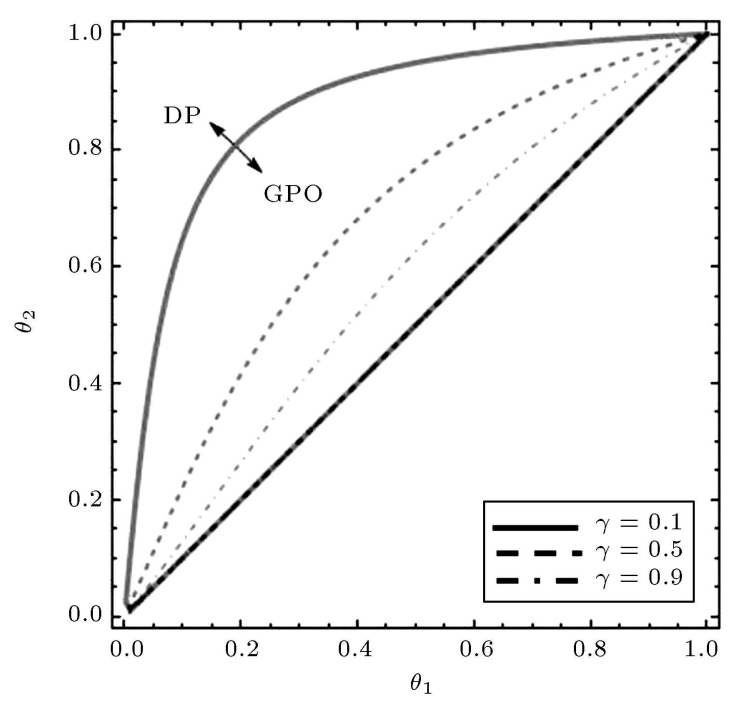

Figure 4. Equilibrium strategy of OEM1 for $\alpha=2$. 


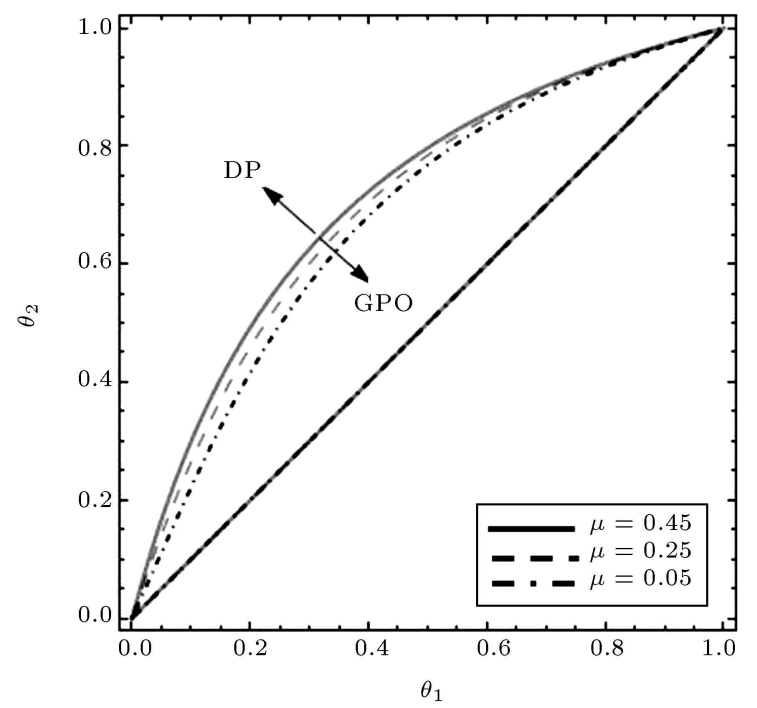

Figure 5. Equilibrium strategy of OEM1 for $\alpha=2$ and $\gamma=0.5$.

It can be concluded from $\tau_{2}$ that discount rate $(\mu)$ has minor effect on it. This is shown in Figure 5.

Because of higher trade surplus under GPO strategy, the size of the pie that must be shared between firms enlarges. This causes a higher centralized profit. Therefore, the GPO's cost advantage, while always benefiting itself, can be a double-edged sword for the competing OEMs (specifically, the weaker one).

Based on this proposition, the stronger OEM has to consider that although it achieves higher profit under GPO strategy, its rival may deviate from GPO when threshold $\tau_{2}$ is not satisfied. Since the stronger OEM achieves less profit through direct procurement by using a GPO (because of deviation of her competitor) rather than its own profit while knowing that it should procure directly from the supplier, the OEM ensures that the threshold is satisfied for implementing the GPO strategy.

In a Stackelberg setting, the GPO is the one that offers wholesale prices to the OEMs. This is the same as $\theta_{2}=\theta_{1}=0$ in the paper. According to Figures 4 and 5 , in the paper (and also substituting $\theta_{2}=\theta_{1}=0$ in the Appendix), it is straightforward to check that both the GPO and DP strategies are equivalent. The intuition for this finding is that, under GPO strategy, the GPO charges the OEMs the same price as they get through the DP strategy from the supply market. This leaves the OEMs with the same profit under DP strategy.

\section{Conclusion}

In this paper, a general model was constructed of competing OEMs with two possible strategies, DP or GPO, given a quantity discount schedule from the manufacturer. Based on linear demand curves, a number of insights into OEMs' group buying decisions were offered when they competed with each other in a common market and had an opportunity to bargain with a GPO to determine the wholesale price. Intuitively, GPO is always advantageous in the absence of competition. Under competition, the symmetric OEMs are always better off using GPO.

Although Group Purchasing Organizations (GPOs) are widely used as an intermediary for group purchasing in a supply chain, and a variety of buyers have benefitted from purchasing cost reductions using GPOs; however, this paper shows that buyers should not use GPO in their purchasing process with closed eyes. Our analysis of OEM-GPO negotiations highlights the strategic perils of group purchasing, and shows that low-cost purchasing GPO may harm OEMs in their profits. This unintuitive result can be explained by different impacts that a GPO exerts on the purchasing process. Although the GPO can enlarge the size of the pie (trade surplus) causing more quantity discount, it affects the slice of the pie (profit sharing) by its bargaining power.

In the symmetric case, the application of the GPO strategy is always the best decision for all supply chain players in equilibrium; however, it is not the case in the asymmetric case. When OEMs are asymmetric, procuring through GPO is not always OEMs' dominant strategy in equilibrium. Moreover, it was shown that an OEM's procurement strategy in equilibrium is dependent not only on its bargaining power but also on its competitor OEM. Interestingly, unlike a weak OEM, a strong OEM (with respect to GPO) may not prefer procuring through GPO. This paper sheds light on vague aspects of purchasing strategy. To expand this even more, investigators may generalize quantity discount function using a general form introduced by Schotanus [21] that describes the underlying function of different discount schedules. Based on this general form, the unit wholesale price, $w(q)$, is:

$$
w(q)=\lambda+\frac{\mu}{q^{e}}
$$

where $\lambda$ is the base price, $\mu$ is the discount scale, and $e$ is the steepness. They show that this QDF fits well with 66 discount schedules found in practice, with $e$ varying from -1.00 to +1.60 . Moreover, researchers may use nonlinear demand functions. In many competitive equilibrium analyses, linear demand functions are often used because of their tractability in providing analytical results. Yet, it is expected to consider demand nonlinearity in many real problems. However, this makes it very difficult to analyze this structure analytically.

Considering two different OEMs in the share of the market (different $\alpha$ 's) or more than two competing OEMs may be other extensions on this research. 


\section{References}

1. Wu, D. "Supply chain intermediation (A Bargaining theoric framework)", In Handbook of Quantitative Supply Chain Analysis, by David Simchi-Levi, David Wu, and Max Shen, pp. 67-115, Kluwer Academic, Dordrecht, Netherlands (2004).

2. Spulber, D.F. "Market microstructure and intermediation", Journal of Economic Perspectives, 10(3), pp. 135-152 (1996).

3. Gans, J.S. "Vertical contracting when competition for orders precedes procurement", The Journal of Industrial Economics (WILEY), 55(2), pp. 325-346 (2007).

4. Ingene, C.A., and Parry, M.E. "Channel coordination when retailers compete", Marketing Science (INFORMS), 14(4), pp. 360-377 (1995).

5. Tsay, A.A. and Agrawal, N. "Channel dynamics under price and service competition", Manufacturing \& Service Operations Management (INFORMS), 2(4), pp. 372-391 (2000).

6. Iyer, G., and Villas-Boas, M.J. "A Bargaining theory of distribution channels", Journal of Marketing Research, 40(1), pp. 80-100 (2003).

7. Dukes, A.J., Gal-Or, E., and Srinivasan, K. "Channel bargaining with retailer asymmetry", Journal of Marketing Research, American Marketing Association, 43(1), pp. 84-97 (2006).

8. Feng, Q. and Lu, L.X. "Supply chain contracting under competition: Bilateral bargaining vs. stackelberg", Production and Operations Management, Production and Operations Management Society, 22(3), pp. 661675 (2013).

9. Feng, Q. and Lu, L.X. "The role of contract negotiation and industry structure in production outsourcing", Production and Operations Management (POMS), 22(5), pp. 1299-1319 (2013).

10. Feng, Q. and Lu, L.X. "The strategic perils of low cost outsourcing", Management Science (INFORMS), 58(6), pp. 1196-1210 (2012).

11. Guo, L. and Iyer, G. "Multilateral bargaining and downstream competition", Marketing Science (INFORMS), 32(3), pp. 411-430 (2013).

12. Jeuland, A. and Shugan, S. "Managing channel profits", Marketing Science (INFORMS), 2(3), pp. 239-272 (1983).

13. Lovejoy, W.S. "Bargaining chains", Management Science (INFORMS), 56(12), pp. 2282-2301 (2010).

14. Chen, R. and Roma, P. "Group buying of competing retailers", Production and Operations Management (POMS), 20(2), pp. 181-197 (2011).

15. Marvel, H.P. and Yang, H. "Group purchasing, nonlinear tariffs, and oligopoly", International Journal of Industrial Organization (Elsevier), 26, pp. 1090-1105 (2008).
16. Hu, Q., and Schwarz, L.B. "Controversial role of GPOs in healthcare-product supply chains", Production and Operations Management (POMS), 20(1), pp. 1-15 (2011).

17. Dana, J.D. "Buyer groups as strategic commitments", Games and Economic Behavior, Elsevier, 74, pp. 470485 (2012).

18. Hu, Q., Schwarz, L.B., and Uhan, N.A. "The impact of group purchasing organizations on healthcare-product supply chains", Manufacturing \& Service Operations Management (INFORMS), 14(1), pp. 7-23 (2012).

19. Stahl, D.O. "Bertrand competition for inputs and Walrasian outcome", American Economic Association, The American Economic Review, 78(1), pp. 189-201 (1988).

20. Gans, J.S. "Vertical contracting when competition for orders precedes procurement", The Journal of Industrial Economics (WILEY), 55(2), pp. 325-346 (2007).

21. Schotanus, F. "Horizontal cooperative purchasing", Ph.D. Dissertation, University of Twente, Enschede (2007).

\section{Appendix: Proofs}

Lemma 1. Using direct procurement, OEMs are the same in equilibrium and GPO is inactive with no profit.

Proof of Lemma 1. The market price is determined based on Relation (1) depending on order quantity $\left(q_{i}\right)$ :

$$
p_{i}=\alpha-q_{i}-\gamma q_{j} .
$$

Under DP strategy, the wholesale price is determined as follows:

$$
w_{i}=w_{s i}=\lambda-\mu q_{i} .
$$

Therefore, the final profit of each OEM is determined based on Relation (A.1):

$$
\pi_{i}=q_{i}\left(p_{i}-w_{i}\right) .
$$

Since each OEM seeks to maximize its own expected profit, it competes in the market based on profit maximization. Each OEM maximizes its gross profit, as shown in Relation (A.1), by choosing order quantities $\left(\max _{q i} \pi_{i}\right)$ which leads to the following first-order conditions:

$$
\begin{gathered}
\frac{\partial \pi_{i}}{\partial q_{i}}=(\alpha-1)-2(1-\mu) q_{i}-\gamma q_{j}=0 \\
\quad(i=1,2 \text { and } j=3-i) .
\end{gathered}
$$

Solving Eq. (A.2) simultaneously for $i=1$ and 2 gives order quantities:

$$
q_{i}=q_{j}=\frac{\alpha-1}{2+\gamma-2 \mu}
$$


Since order quantities of both OEMs are the same, other variables are the same too:

$$
\begin{aligned}
& p_{i}=p_{j}=\frac{1+\alpha+\gamma-2 \alpha \mu}{2+\gamma-2 \mu} \\
& w_{i}=w_{j}=1-\frac{\mu(\alpha-1)}{2+\gamma-2 \mu}, \\
& \pi_{i}=\pi_{j}=\frac{(\alpha-1)^{2}(1-\mu)}{(2+\gamma-2 \mu)^{2}}
\end{aligned}
$$

Since GPO has no role in DP strategy, it is clear that he/she may not receive any profit.

Lemma 2. Using GPO strategy, the most important variables (order quantity and wholesale price) are as follows:

$$
\begin{aligned}
q_{i}= & \frac{\left(\xi(1-\alpha)\left(\xi(2-\gamma)-2 \mu \kappa_{i}\right)\right)}{\eta} \\
w_{i}= & w_{c i}=\frac{1}{\eta}\left(\left(\gamma^{2}-4+\mu(2+6 \alpha-4 \alpha \mu-\gamma-3 \alpha \gamma)\right) \xi^{2}\right. \\
& +2 \mu(2-2 \alpha+\alpha \mu+\alpha \gamma-\mu) \kappa_{i} \xi+2 \mu \gamma \kappa_{j} \xi \\
& \left.+4 \alpha \mu^{2} \kappa_{i} \kappa_{j}(1-\xi)\right)
\end{aligned}
$$

Proof of Lemma 2. We define $\theta_{i}$ and $k_{i}$ as the bargaining power of OEM $(i)$ and GPO in a bilateral negotiation, respectively, and we have $\theta_{i}=1-\kappa_{i}$. For simplicity, parameters $\xi$ and $\eta$ are defined as follows:

$$
\begin{aligned}
& \xi=\kappa_{i}+\kappa_{j}-\kappa_{i} \kappa_{j}, \\
& \eta=\xi^{2}\left(\gamma^{2}-4(\mu-1)^{2}-2 \mu \gamma\right)+2 \mu \kappa_{i} \kappa_{j}(\gamma \xi+2 \mu(1-\xi)) .
\end{aligned}
$$

Since an "early ordering" approach is applied in procurement strategy, OEMs compete in the market firstly to determine their order quantities and then bargain bilaterally with the GPO to determine the wholesale price they should pay based on Nash bargaining solution. Here, there is a sub-game perfect equilibrium and a backward induction approach is used to determine two main variables for each OEM.

At the first step, we consider order quantities as given and try to determine "wholesale prices" in the bargaining stage. For this and according to the Nashbargaining solution approach, the wholesale prices are determined by simultaneously maximizing Nashproduct relation pre-defined in Relation (3) for $i=1,2$ and $j=3-i$ :

$$
w_{c i}=\frac{1-\mu q_{i}-\theta_{i}\left(\theta_{j}+2 \mu q_{j}-\mu \theta_{j} q_{i}-2 \mu \theta_{j} q_{j}\right)}{1-\theta_{i} \theta_{j}} .
$$

It is worth commenting that the profit of GPO $\left(\pi_{c}\right)$ is defined as follows:

$$
\pi_{c}=q_{1}\left(w_{c 1}-w_{s c}\right)+q_{2}\left(w_{c 2}-w_{s c}\right),
$$

where $q_{1}$ and $q_{2}$ are given; $w_{c i}$ is going to be determined; and $w_{s c}$ is the wholesale price that GPO pays to supplier based on quantity discount schedule:

$$
w_{s c}=\lambda-\mu\left(q_{1}+q_{2}\right) .
$$

In addition, the reservation profits in Relation (3) are determined as follows:

$$
\begin{aligned}
& \delta_{i}=q_{i}\left(p_{i}-w_{s i}\right), \\
& \delta_{c i}=0 .
\end{aligned}
$$

Relation (A.14) states that OEM (i) can directly procure her committed orders from the supplier when bilateral negotiation with GPO fails (disagreement point). In addition, if there is no agreement between $\operatorname{OEM}(i)$ and GPO, then GPO can just contract with OEM $(i)$ 's competitor. However, since there is no advantage for $\operatorname{OEM}(j)$ in using $\operatorname{GPO}\left(q_{c}=q_{j} \Rightarrow w_{s j}=\right.$ $\left.w_{s c}\right)$, GPO's reservation profit becomes zero.

In the second step, OEMs compete in the market to determine their order quantities based on maximizing their gross profits shown in Relation (A.1). In this regard, the following first-order conditions should be solved simultaneously:

$$
\left(\frac{\partial \pi_{i}}{\partial q_{i}}=0 ; \frac{\partial \pi_{j}}{\partial q_{j}}=0\right) \quad\left(i=1,2 \text { and } \begin{array}{r}
j= \\
(\text { A.16) }
\end{array}\right.
$$

This leads to Eq. (A.17) as shown in Box A.I. By using $\kappa_{i}, \xi$, and $\eta$, as defined previously, Relation (A.17) is simplified to Relation (A.7). After substituting $q_{i}$ and $q_{j}$ in Relation (A.11) and doing some simplifications, we reach Relation (A.8) and the proof is completed.

According to Relations (1) and (A.7), we can get the market prices by Eq. (A.18) as shown in Box A.II. When Relations (A.9), (A.17), and (A.18)

$$
q_{i}=\frac{\left((1-\alpha)\left(1-\theta_{i} \theta_{j}\right)\left((2-\gamma)\left(1-\theta_{i} \theta_{j}\right)-2\left(1-\theta_{i}\right) \mu\right)\right)}{\left(\left(-4+\gamma^{2}\right)\left(1-\theta_{i} \theta_{j}\right)^{2}+2\left(1-\theta_{i} \theta_{j}\right)\left(4-4 \theta_{i} \theta_{j}-\gamma\left(\theta_{j}+\theta_{i}\left(1-2 \theta_{j}\right)\right)\right) \mu-4\left(1-\theta_{i} \theta_{j}\left(3-\theta_{i}-\theta_{j}\right)\right) \mu^{2}\right)}
$$




$$
p_{i}=\frac{2 \mu \xi\left(\gamma\left(\alpha \kappa_{i}+\kappa_{j}\right)-(\alpha-1) \kappa_{i}\right)-\xi^{2}\left((2-\gamma)(1+\alpha+\gamma-4 \alpha \mu)+4 \alpha \mu^{2}\right)+4 \alpha \mu^{2} \kappa_{i} \kappa_{j}(1-\xi)}{\eta}
$$

Box A.II

$$
\begin{aligned}
& \pi_{i}=\frac{\xi^{2}(\alpha-1)^{2}(1-\mu)\left(2 \mu \kappa_{i}-(2-\gamma) \xi\right)^{2}}{\eta^{2}} ; \quad(i=1,2 \quad \& \quad j=3-i), \\
& \pi_{c}=\frac{2 \kappa_{i} \kappa_{j} \xi(\alpha-1)^{2} \mu\left((2-\gamma)^{2} \xi^{2}-2 \mu(2-\gamma)\left(\xi^{2}+\kappa_{i} \kappa_{j}\left(\xi-\frac{2 \mu}{2-\gamma}\right)\right)\right)}{\eta^{2}} .
\end{aligned}
$$

are substituted in Relation (A.1), then firms' profits are derived by Eqs. (A.19) and (A.20) as shown in Box A.III.

Lemma 3 (feasible conditions). Besides some general conditions for parameters, there is always a unique upper bound for " $\alpha$ " under different procurement strategies. The feasible area under DP is always larger than that under GPO.

Proof of Lemma 3. We have some general conditions for parameters that are held in the real world (as explained in Section 3, note that forcing $\theta_{1} \leq \theta_{2}$ is just an assumption we made in this paper):

$$
\begin{aligned}
& 0<\gamma<1, \quad 0<\mu<\frac{1}{2}, \quad 1<\alpha, \\
& 0<\theta_{1} \leq \theta_{2}<1
\end{aligned}
$$

Besides these general conditions, all problem variables (order quantities, market prices, wholesale prices, and firms' profits) must be positive, too.

When OEMs procure directly from the supplier, according to general conditions (A.21), Relations (A.3), (A.4), and (A.6) in Lemma 1 are always positive. However, making wholesale prices based on Relation (A.5) positive needs an extra condition:

$$
1<\alpha \leq \frac{2+\gamma-\mu}{\mu}
$$

$u \alpha^{\mathrm{DP}}=\frac{2+\gamma-\mu}{\mu}$ is an upper bound for $\alpha$ under DP strategy. This unique upper bound increases in $\gamma$ and decreases in $\mu$.

Procuring OEMs through GPO concludes the following.

According to general conditions (A.21) and comparing $p_{i}^{\mathrm{GPO}}$ with $w_{c i}^{\mathrm{GPO}}$ (Relations (A.11) and (A.18)), we understand that $p_{i}^{\mathrm{GPO}}>0$ is dominated by $w_{c i}^{\mathrm{GPO}}>$ 0 . Therefore, we just need to consider $w_{c i}^{\mathrm{GPO}}>0$.
$\pi_{i}^{\mathrm{GPO}}$ is always positive for both OEMs and $\pi_{c}^{\mathrm{GPO}}>0$ is dominated by $q_{i}^{\mathrm{GPO}}>0$.

Therefore, under GPO strategy, $q_{i}^{\mathrm{GPO}}>0$ and $w_{c i}^{\mathrm{GPO}}>0$ are sufficient conditions. $q_{i}^{\mathrm{GPO}}>0$ is always positive. Considering $w_{c i}^{\mathrm{GPO}}>0$, we would have two

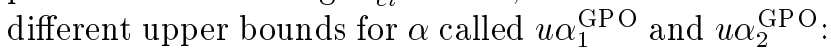

$$
\begin{aligned}
& u \alpha_{1}^{\mathrm{GPO}} \\
& =\frac{\left(\gamma^{2}-4+2 \mu-\gamma \mu\right) \xi^{2}+2 \mu(2-\mu) \kappa_{1} \xi+2 \gamma \mu \kappa_{2} \xi}{3 \mu(\gamma-2) \xi^{2}+2 \mu(\mu-\gamma+2) \kappa_{1} \xi+4 \mu^{2} \kappa_{2}\left(\xi-\kappa_{1}\right)},
\end{aligned}
$$

$u \alpha_{2}^{\mathrm{GPO}}$

$$
=\frac{\left(\gamma^{2}-4+2 \mu-\gamma \mu\right) \xi^{2}+2 \mu(2-\mu) \kappa_{2} \xi+2 \gamma \mu \kappa_{1} \xi}{3 \mu(\gamma-2) \xi^{2}+2 \mu(\mu-\gamma+2) \kappa_{2} \xi+4 \mu^{2} \kappa_{1}\left(\xi-\kappa_{2}\right)}
$$

As $\theta_{1} \leq \theta_{2}$ and $\kappa_{2} \leq \kappa_{1}$, accordingly. Then, it is obvious that $u \alpha_{2}^{\mathrm{GPO}} \leq u \alpha_{1}^{\mathrm{GPO}}$. Thus, we need to just consider $u \alpha_{2}^{\mathrm{GPO}}$ as the upper bound for $\alpha$, and $u \alpha^{\mathrm{GPO}}=u \alpha_{2}^{\mathrm{GPO}}$ is the unique upper bound for $\alpha$ under GPO strategy.

By comparing upper bounds for $\alpha$ under two different procurement strategies, we get $u \alpha^{\mathrm{GPO}} \leq$ $u \alpha^{\mathrm{DP}}$. It means that the feasible area under DP is always larger than that under GPO.

Proof of Proposition 1. The proof for the GPO is straightforward. The GPO is out of the trade under DP strategy; therefore, it receives no profit. However, he has positive profit under GPO strategy. We define $\rho=2+\gamma-2 \mu$; thus, based on Relation (A.20), the gross profit for GPO in the symmetric case is as follows:

$$
\pi_{c}^{\mathrm{GPO}}=\frac{2 \mu(\alpha-1)^{2}\left(1-\theta^{2}\right)}{(\rho+(\rho-2 \mu) \theta)^{2}} .
$$

The above term is always positive (under the general conditions described in Lemma 3 ), except when $\theta=1$, 
meaning that OEMs have all bargaining power and get the whole trade surplus under negotiation (we know that GPO's reservation profit is zero) with no profit for GPO.

Simplifying Relation (A.19) for the symmetric case, OEMs' profit is obtained as follows:

$$
\pi_{i}^{\mathrm{GPO}}=\frac{(1-\mu)(\alpha-1)^{2}(1+\theta)^{2}}{(\rho+(\rho-2 \mu) \theta)^{2}} .
$$

Based on the comparison of Relations (A.6) and (A.26), it is simply proved that always $\pi_{i}^{\mathrm{GPO}} \geq \pi_{i}^{\mathrm{DP}}$.

Proof of Proposition 2. According to Relations (A.7), (A.8), and (A.18)-(A.20), if we differentiate them with respect to $\theta$, we have:

$$
\begin{array}{ll}
\frac{\partial p_{i}}{\partial \theta} \leq 0, & \frac{\partial q_{i}}{\partial \theta} \geq 0, \\
\frac{\partial \pi_{i}}{\partial \theta} \geq 0, & \frac{\partial \pi_{c}}{\partial \theta} \leq 0 .
\end{array}
$$

They show the results in Proposition 2.

Proof of Proposition 3. According to Relations (A.7), (A.8), and (A.19)-(A.20), if we differentiate them with respect to $\gamma$, we have:

$$
\frac{\partial q_{i}}{\partial \gamma} \leq 0, \quad \frac{\partial w_{c i}}{\partial \gamma} \geq 0, \quad \frac{\partial \pi_{i}}{\partial \gamma} \leq 0, \quad \frac{\partial \pi_{c}}{\partial \gamma} \leq 0 .
$$

They show the results in Proposition 2.

However, the behavior of market price with respect to $\gamma$ is different and depends on other parameters. $\frac{\partial p_{i}}{\partial \gamma}$ is negative most of the time; therefore, the market price usually decreases in $\gamma$. In the case of $\frac{1}{4}<\mu<\frac{1}{2}$, we have a threshold $\theta<\frac{1-2 \mu}{4 \mu-1}$ (called $\tau 1$ ). It means that if $\theta>\tau 1$, then market price increases in $\gamma$.

Proof of Proposition 4. According to Relations (A.7), (A.8), and (A.18)-(A.20), if we differentiate them with respect to $\mu$, we have:

$$
\begin{array}{ll}
\frac{\partial p_{i}}{\partial \mu} \leq 0, & \frac{\partial q_{i}}{\partial \mu} \geq 0, \\
\frac{\partial \pi_{i}}{\partial \mu} \geq 0, & \frac{\partial \pi_{c}}{\partial \mu} \geq 0 .
\end{array}
$$

They show the results in Proposition 4.

Proof of Proposition 5. When procuring directly from supplier, OEMs are equal in all parameters; the only difference between them appears when procuring through GPO. Therefore, it is clear that they have identical behavior under DP strategy, and all variables are derived according to Lemma 1.

When procuring through GPO, all variables are derived according to Lemma 2. All results presented in Proposition 5 are simply proved based on general the conditions presented in Relation (A.21).

\section{Proof of Proposition 6.}

GPO: It is clear that the GPO always benefits from group purchasing strategy because it obtains zero profit under DP strategy.

OEM2: For OEM2, we have to prove that $\pi_{2}^{\mathrm{GPO}}>$ $\pi_{2}^{\text {DP }}$. From (A.6) and (A.19), we have:

$$
\begin{aligned}
\pi_{2}^{\mathrm{GPO}} & >\pi_{2}^{\mathrm{DP}} \rightarrow \\
& \frac{\xi^{2}(\alpha-1)^{2}(1-\mu)\left(2 \mu \kappa_{2}-(2-\gamma) \xi\right)^{2}}{\eta^{2}} \\
& >\frac{(\alpha-1)^{2}(1-\mu)}{(2+\gamma-2 \mu)^{2}} \rightarrow \\
& \frac{\xi^{2}\left(2 \mu \kappa_{2}-(2-\gamma) \xi\right)^{2}}{\eta^{2}} \\
& >\frac{1}{(2+\gamma-2 \mu)^{2}} \rightarrow \\
& \frac{\left(\xi^{2}\left(2 \mu \kappa_{2}-(2-\gamma) \xi\right)^{2}\right)(2+\gamma-2 \mu)^{2}-\eta^{2}}{\eta^{2}(2+\gamma-2 \mu)^{2}}>0 \rightarrow \\
& \xi^{2}\left(2 \mu \kappa_{2}-(2-\gamma) \xi\right)^{2}(2+\gamma-2 \mu)^{2}-\eta^{2}>0 \rightarrow \\
& \left(\xi\left(2 \mu \kappa_{2}-(2-\gamma) \xi\right)(2+\gamma-2 \mu)-\eta\right)\left(\xi \left(2 \mu \kappa_{2}\right.\right. \\
& -(2-\gamma) \xi)(2+\gamma-2 \mu)+\eta)>0 .
\end{aligned}
$$

Right hand side of Relation (A.27) consists of two parts:

$$
\begin{aligned}
& \text { (a) }\left(\xi\left(2 \mu \kappa_{2}-(2-\gamma) \xi\right)(2+\gamma-2 \mu)+\eta\right) \text {, } \\
& \text { (b) }\left(\xi\left(2 \mu \kappa_{2}-(2-\gamma) \xi\right)(2+\gamma-2 \mu)-\eta\right) \text {. }
\end{aligned}
$$

As is known, $\eta<0$ under the conditions prescribed in Lemma 3. We can simply show that $\left(2 \mu \kappa_{2}-(2-\gamma) \xi\right)<$ 0 . Since $\xi$ and $(2+\gamma-2 \mu)$ are always positive, part (a) is always negative.

If we prove that part (b) is negative too, then it is proved that $\pi_{2}^{\mathrm{GPO}}>\pi_{2}^{\mathrm{DP}}$. Now, we focus on part (b):

$$
\begin{gathered}
\left(\xi\left(2 \mu \kappa_{2}-(2-\gamma) \xi\right)(2+\gamma-2 \mu)-\eta\right)<0 \rightarrow \\
\xi\left(2 \mu \kappa_{i}-(2-\gamma) \xi\right)(2+\gamma-2 \mu)<\eta .
\end{gathered}
$$

It can simply be shown that, under feasible conditions (Lemma 3), it is true. Therefore, the proposition for OEM2 is proved.

OEM1: We perform a similar procedure for OEM1. To prove that $\pi_{1}^{\mathrm{GPO}}>\pi_{1}^{\mathrm{DP}}$, we have a relation similar to Eq. (A.27): 


$$
\begin{aligned}
& \left(\xi\left(2 \mu \kappa_{1}-(2-\gamma) \xi\right)(2+\gamma-2 \mu)-\eta\right) \\
& \quad\left(\xi\left(2 \mu \kappa_{1}-(2-\gamma) \xi\right)(2+\gamma-2 \mu)+\eta\right)>0 .
\end{aligned}
$$

Left hand side of Relation (A.30) consists of two parts:

(a) $\left(\xi\left(2 \mu \kappa_{1}-(2-\gamma) \xi\right)(2+\gamma-2 \mu)+\eta\right)$

(b) $\left(\xi\left(2 \mu \kappa_{1}-(2-\gamma) \xi\right)(2+\gamma-2 \mu)-\eta\right)$,

As mentioned before (proof of OEM2), we can simply show that part (a) is always negative. Therefore, OEM1 prefers GPO to DP strategy if and only if part (b) is negative. Based on the definition of $\eta$ (Lemma 2), part (b) is shown as:

$$
\begin{aligned}
& \xi(2+\gamma-2 \mu)\left(\xi(-2+\gamma)+2 \kappa_{1} \mu\right) \\
& \quad-\left(\xi^{2}\left(\gamma^{2}-4(-1+\mu)^{2}-2 \gamma \mu\right)\right. \\
& \left.\quad+2 \kappa_{1} \kappa_{2} \mu\left(\xi \gamma+2\left(-1+\kappa_{1}\right)\left(-1+\kappa_{2}\right) \mu\right)\right) .
\end{aligned}
$$

Being negative of part (b), as $\xi>0$, then:

$$
\begin{aligned}
& \xi \kappa_{1}\left(2+\gamma-\gamma \kappa_{2}-2 \mu\right)+2 x i^{2}(-1+\mu) \\
& +2 \kappa_{1} \kappa_{2}(-1+\xi) \mu<0 \rightarrow(2 \xi(-1+\mu) \\
& \left.+\kappa_{1}\left(2+\gamma-\gamma \kappa_{2}+2\left(-1+\kappa_{2}\right) \mu\right)\right)<2 \frac{\kappa_{1} \kappa_{2}}{\xi} \mu \rightarrow \\
& \left(2 \frac{\xi}{\kappa_{1} \kappa_{2}}(-1+\mu)+(2+\gamma-2 \mu) \frac{1}{\kappa_{2}}-(\gamma-2 \mu)\right)<2 \frac{\mu}{\xi} \rightarrow \\
& \left((2-\gamma)-2(1-\mu) \frac{1}{\kappa_{1}}+\gamma \frac{1}{\kappa_{2}}\right)<2 \frac{\mu}{\xi} \rightarrow \\
& \left((2-\gamma) \xi-2(1-\mu)\left(1+\frac{\kappa_{2}}{\kappa_{1}}-\kappa_{2}\right)\right. \\
& \left.+\gamma\left(1+\frac{\kappa_{1}}{\kappa_{2}}-\kappa_{1}\right)\right)<2 \mu \rightarrow \\
& \left((2-\gamma)(\xi-1)+\gamma\left(\frac{\kappa_{1}}{\kappa_{2}}-\kappa_{1}\right)\right. \\
& \left.-2(1-\mu)\left(\frac{\kappa_{2}}{\kappa_{1}}-\kappa_{2}\right)\right)<0 \rightarrow \\
& \left(\gamma \frac{\kappa_{1}}{\kappa_{2}}-2(1-\mu)\left(\frac{\kappa_{2}}{\kappa_{1}}\right)\left(\frac{1-\kappa_{1}}{1-\kappa_{2}}\right)\right)
\end{aligned}
$$

$$
\begin{aligned}
& -(2-\gamma)\left(1-\kappa_{1}\right)<0 \rightarrow \\
& \gamma \frac{1-\theta_{1} \theta_{2}}{1-\theta_{2}}-2(1-\mu)\left(\frac{1-\theta_{2}}{1-\theta_{1}}\right)\left(\frac{\theta_{1}}{\theta_{2}}\right)-2 \theta_{1}<0 .
\end{aligned}
$$

We define a threshold:

$$
\begin{aligned}
\tau_{2}= & 2(1-\mu)\left(\frac{1-\theta_{2}}{1-\theta_{1}}\right)\left(\frac{\theta_{1}}{\theta_{2}}\right)+2 \theta_{1} \\
& -\gamma \frac{1-\theta_{1} \theta_{2}}{1-\theta_{2}}
\end{aligned}
$$

Whenever $\tau_{2}>0$, then $\pi_{1}^{\mathrm{GPO}}>\pi_{1}^{\mathrm{DP}}$, and vice versa. $\square$

\section{Biographies}

Mojtaba Soleimani Sedehi is a $\mathrm{PhD}$ candidate at the Department of Industrial Engineering at Iran University of Science and Technology. His main research focus is on logistics and supply chain management. He has published 9 books and more than 15 journal and conference papers in this area; in addition, he has done 12 research projects mostly for the "Ministry of Industry, Mine and Trade" of Iran during his 13 years of research experience. In addition, he is the Editor-in-Chief of "Logistics and Supply Chain" journal published monthly in Iran.

Ahmad Makui is a Professor at the School of Industrial Engineering in Iran University of Science and Technology. He has received his $\mathrm{PhD}$ in Industrial Engineering from Iran University of Science and Technology. His researches are on operations research and decision-making. His works focus also on competitive supply chain and forward and reverse logistics. He has more than one hundred papers in various journals.

Ehsan Bolandifar is an Assistant Professor at the Chinese University of Hong Kong. His research mainly focuses on the operations management interfaces with finance and marketing. $\mathrm{He}$ is also interested in component procurement in supply chains, and his research has been featured in top-tier journals on operations management such as Manufacturing and Service Operations Management and Production and Operations Management. Professor Bolandifar has received his $\mathrm{PhD}$ in Business Administration from Washington University in St. Louis and his MSc from Sharif University of Technology. 